Communications in Physics, Vol. 25, No. 1 (2015), pp. 91-96

DOI:10.15625/0868-3166/25/1/5661

\title{
SIMULATION FOR NEUTRON TRANSPORT IN PWR REACTOR MODERATOR AND EVALUATION FOR PROPER THICKNESS OF LIGHT WATER REFLECTOR
}

\author{
NGUYEN TUAN KHAI AND PHAN QUOC VUONG \\ Institute for Nuclear Science and Technology, 179 Hoang Quoc Viet, Hanoi \\ E-mail: ntkhai@iop.vast.ac.vn
}

Received 26 November 2014

Accepted for publication 12 December 2014

\begin{abstract}
Monte Carlo calculation method can be used for resolving particle transport in matter, and particularly the transport of neutrons in the environment of the reactor core. The method has become more efficient because of high accuracy of updated nuclear data and fast development of advanced super-computing system. In this work, we would like to present calculations for kinematic characteristics of neutron transport in a typical configuration of the pressurized water reactor $(P W R)$ fuel assembly based on the Monte-Carlo simulation method. We concentrate in two main results: (1) neutron energy spectrum at fuel rod and (2) optimal thickness of light water reflector.
\end{abstract}

Keywords: Monte-Carlo method, neutron transport, pressurized water reactor (PWR), fuel rod, fuel assembly, light water reflector.

\section{INTRODUCTION}

Fission reaction takes place when a heavy nucleus as uranium, thorium and plutonium absorbs neutron, high energy gamma radiation or even charged particles and is splited into two intermediate-mass fragments with releasing a few new neutrons under prompt and delayed types. As known, amongst of these the fission of the heavy actinide isotopes as ${ }^{235} \mathrm{U},{ }^{239} \mathrm{U}$ and ${ }^{241} \mathrm{Pu}$ induced by thermal neutrons is the most important process to generate power in nuclear reactor. It is obvious that in nuclear reactor the neutron generation from fission and their transport including slowing down in moderator and absorption in fuel are very essential as basis of reactor physics and kinetics.

The basis of transport theory can be derived from the particle density balance inside an infinitesimal element of the six-dimensional phase-space. In the Cartesian geometry space, the particles are initially located inside an infinitesimal volume element $d^{3} r=d x d y d z$ about position $r$. The infinitesimal element in the angular space is the differential solid angle $d \Omega$ at the direction of motion $\Omega$. The energies of the particles are simply distributed within energy interval from $E$ to $E+d E$. An illustration is given in Fig. 1.

(C)2015 Vietnam Academy of Science and Technology 
The neutron balance inside the six-dimensional phase-space element is described by the transport equation [1]:

$$
(1 / v) \partial[\psi(r, \Omega, E, t)] / \partial t+\Omega \cdot \nabla \psi(r, \Omega, E, t)+\Sigma_{t}(r, E) \psi(r, \Omega, E, t)=q(r, \Omega, E, t)
$$

where $\psi(r, \Omega, E, t)$ is the neutron flux, $\Sigma_{t}(r, E)$ the total macroscopic cross section of the medium and $q(r, \Omega, E, t)$ a generalized source term. The first term on the left-hand side is the time rate of change in the neutron density. The second term describes the rate at which neutrons travelling in the direction of motion $\Omega$ leave or enter the differential volume element $d^{3} r$. The third term consists of all interactions removing neutrons either by absorption or scattering away from the differential angular and energy space. The source term $q(r, \Omega, E, t)$ consists of contribution from the external, scattering and fission sources. If the source and loss terms are in balance, the time dependence disappears, and the result is the steady-state

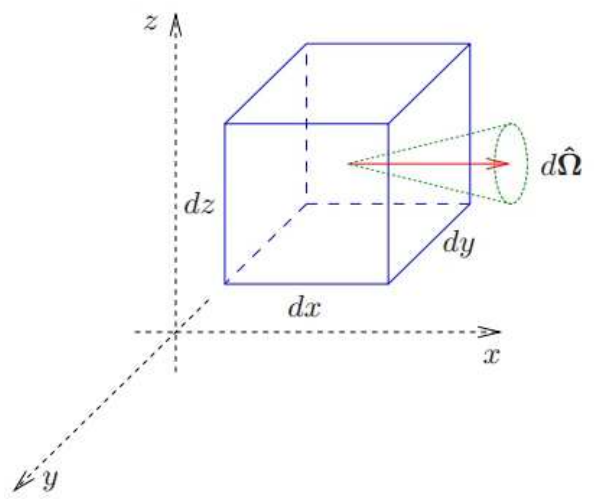

Fig. 1. A moving particle located inside an infinitesimal volume element $d^{3} r$, where the energy of the particle lies within interval $d E$ at a given value $E$ transport equation.

In this work we have used the Monte Carlo (MC) calculations for resolving neutron transport problem in the light water moderator for the PWR fuel assembly based on the physical processes described by Eq. (1). The MC method is being widely used in nuclear physics, radiation physics and shielding calculations. The basic principle of the method is not so complicated. The life of a single neutron or any other particle type is simulated from its initial emission until the eventual death by absorption in the system or escape from the defined boundaries of the system. The frequency and outcome of the various interactions that may occur during the particle's life are randomly sampled and simulated according to interaction laws derived from particle physics. When the procedure is repeated for a large number of particles, the obtained result shows a detailed simulation of the transport process. In reactor physics applications, the method has been developed to solve the neutron transport problem, consider neutronic characteristics and analyze criticality safety in the reactor core. These researches play role as physics core in the present MC computation codes such as MCNP, MVP/GMVP. The main advantage of the MC method is a good adaptability in modeling geometry and interaction physics without any approximations. In practice, the method is possible to include with relative ease all desired variables, which may be three spatial coordinates, energy, two directional variables and time, and to represent accurately almost any physical model for particle scattering, cross section data and geometric detail. Recently, the application of the Monte Carlo method has become more efficient because of high accuracy of updated nuclear data and fast development of advanced super-computing system.

We have constructed the MC calculation algorithms to simulate the neutron transport in a typical configuration of the PWR fuel assembly, where it is assumed that the neutrons are generated from the fission of ${ }^{235} \mathrm{U}$ in fuel rods and considered until they are either absorbed in the moderator, or hit on the fuel rods. The interactions of neutron inside the fuel are not considered 
here. In addition, it is also assumed that the reactor core is surrounded by a light water reflector, then the trajectory of the neutrons hitting boundary between the core and the reflector are continued to be followed. Based on considering a quantity called "backscattering fraction", i.e. the ratio between the neutrons coming back the core from the reflector to the total number of the neutrons hitting the boundary, as a function of the reflector thickness, the optimization for the thickness of the light water reflector is evaluated.

\section{CALCULATION PROCEDURE}

Fig. 2 shows the typical configuration of the PWR fuel assembly. The dimension of the assembly is $21.4 \times 21.4 \mathrm{~cm}^{2}$ containing 289 rods $(17 \times 17)$ including 264 fuel rods, 24 control rods and one instrumental guide tube. A coordinate $\mathrm{XYZ}$ is established with the origin $\mathrm{O}$ at center of the assembly. This made us possible to define the position of the rods in the assembly and to track the neutron trajectory during transport.

The fission energy spectrum of ${ }^{235} \mathrm{U}$ is shown in Fig. 3 and expressed by [3]:

$$
P_{n}(E)=0.453 e^{-1.036 E} \sinh \sqrt{ } 2.29 E
$$

where $P_{n}(E)$ is probability for generation of neutrons with energy from $E$ to $E+d E$.

The MC simulation calculations have been carried out by tracking the transport of neutron in the moderator (light water) and the position at which the neutron hits the fuel rods. The neutrons are assumed to be emitted from the fission source as described by expression (2). Then, the life of these neutrons from their birth to the death, which is referred as the particle history, is considered based on the statistical nature of three main interaction processes: elastic and inelastic scattering, and the radiation capture reactions. In our calculations a set of neutron histories is generated by following individual neutrons through successive collisions. The locations and results of collisions are determined from the range of probabilities by sets of random numbers. The simulation procedure is summarized as follows:

- As mentioned above, the fuel rods are assumed to be the sources to generate the fission neutrons with the energy spectrum shown in Fig. 3. Three initial parameters related to the neutron emission which are the direction, energy and position are generated as random variables.

- Then, the distance the neutron travels from the emitted position on the source to its first

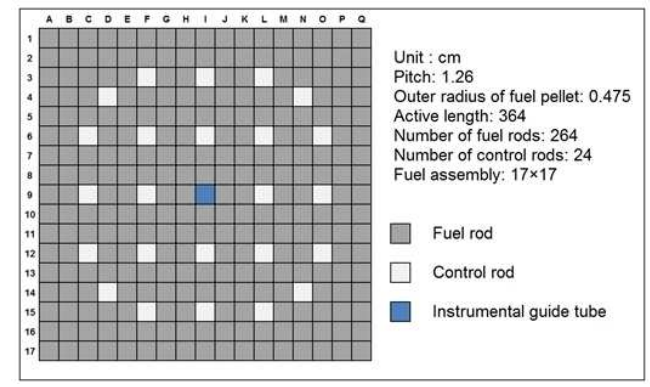

Fig. 2. Typical configuration of the PWR fuel assembly [2]

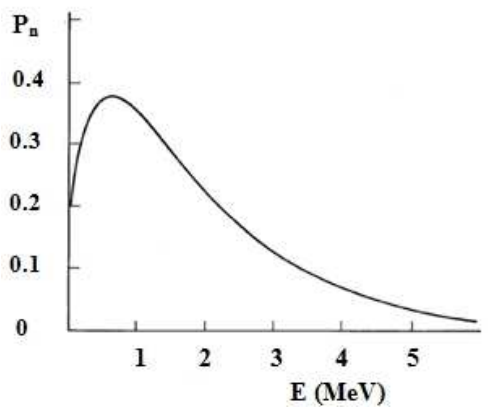

Fig. 3. Neutron fission spectrum of ${ }^{235} \mathrm{U}$ 
interaction is defined as free path by random sampling:

$$
\lambda=-\ln (r) / \Sigma_{T}
$$

where $\Sigma_{T}$ is total macroscopic cross section,

$$
\Sigma_{T}=\Sigma_{T-H}+\Sigma_{T-O}
$$

with $\Sigma_{T-H}$ and $\Sigma_{T-O}$ being the total macroscopic cross sections for hydrogen and oxygen, respectively, in which the main interaction processes mentioned above, i.e. elastic and inelastic scattering, and radiation capture (absorption), are taken into account: $\mathrm{p}\left(\mathrm{n}, \mathrm{n}^{\prime}\right) \mathrm{p},{ }^{16} \mathrm{O}\left(\mathrm{n}, \mathrm{n}^{\prime}\right){ }^{16} \mathrm{O}$, ${ }^{16} \mathrm{O}\left(\mathrm{n}, \mathrm{n}^{\prime}\right){ }^{16} \mathrm{O}^{*}$ with producing $6.1 \mathrm{MeV} \gamma$-ray and radiation capture $\mathrm{p}(\mathrm{n}, \gamma) \mathrm{d}$ with producing 2.2 $\mathrm{MeV} \gamma$-ray.

- At a given interaction position, the atom of the material with which the neutron interacts and the type of interaction are considered via random sampling based on the normalized probability distributions built from the cross section data of the interactions of interest. If the interaction leads to absorption, the life of neutron is terminated. If the interaction is elastic scattering or inelastic scattering, the direction of scattered neutron is selected from the distribution of scattering angles, i.e. differential cross section, and the energy of the scattered neutron is determined using energy and momentum conservations.

- To follow the neutron trajectory in the original coordinate a series of frame rotations has to be done after each collision in order to convert the neutron's position from the existing coordinate into the original one. This is necessary to determine the physical parameters characteristics for the slowing down process such as moderation length, number of collisions and etc.

In this work we have used a large library of nuclear data on cross section and angular distribution from National Nuclear Data Center, Brookhaven Laboratory, US [4]. They are necessary to build the normalized probability distributions for the MC random sampling algorithm.

\section{RESULTS AND DISCUSSION}

First of all, to validate the simulation algorithms we have performed two calculations for comparison:

Firstly, the conventional calculations related to the neutron transport and moderation including: (1) the energy decrease of $2 \mathrm{MeV}$ neutrons as a function of the number of collisions in hydrogen and oxygen (the calculation results are shown in Fig. 4), and (2) the collision-number distribution for neutron slowing down from $2 \mathrm{MeV}$ energy to the thermal one in Hydrogen (the obtained distribution is shown in Fig. 5). Our obtained results from these calculations are well consistent with the well-known references $[5,6]$.

Secondly, based on the configuration of PWR fuel assembly (shown in Fig. 2) it is assumed that a neutron source placed at the center of the assembly generates the mono-energy neutrons of $2 \mathrm{MeV}$ we have compared the results obtained by our calculation program and MCNP code for both the neutron energy and flux spectra along the assembly. Figs. $6 \& 7$ show a good consistence for these comparisons.

Thus, we can conclude on validation for the used nuclear data and simulation algorithms. Following, the calculations were used to consider the neutron transport in the PWR fuel assembly, where the fission neutron source is generated at the fuel rods. 


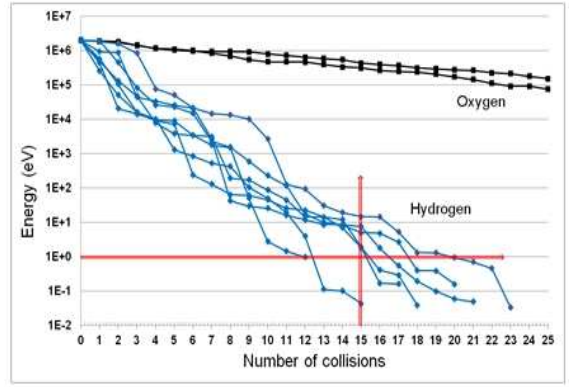

Fig. 4. Energy decrease of $2 \mathrm{MeV}$ neutrons as a function of collisions in Hydrogen and Oxygen

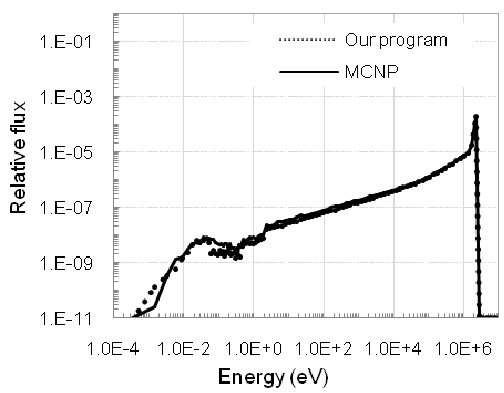

Fig. 6. Neutron energy spectrum at the fuel rod using a mono-energy neutron source of $2 \mathrm{MeV}$.

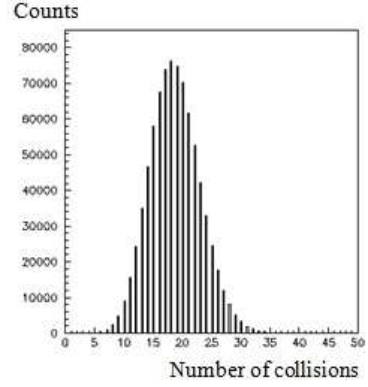

Fig. 5. Collision number distribution for 2 $\mathrm{MeV}$ neutrons slowing down to thermal energy in Hydrogen

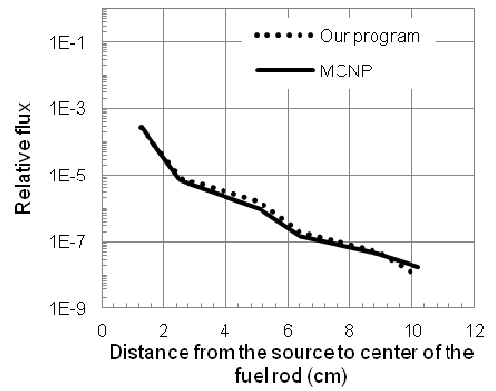

Fig. 7. Neutron flux as a function of distance from the source to the fuel rod.

The first result we have obtained is the energy spectrum of the neutrons hitting the fuel rods and core boundaries. Fig. 8 shows typical spectrum at one rod. In principle, the registered neutron amount with energies from $E$ to $E+d E$ depends on the position at which neutron hits the rod and also the position of the rods in the assembly.

A gross spectrum at each assembly can be obtained by summing the spectral data from all the rods. This makes us possible to determine two essential distributions on the neutron flux, particularly for thermal neutrons: neutron distribution along each assembly and neutron distribution between the assemblies in the core. Physically, two these results will give image of power distribution in the reactor core. However, in frame of this work we only concentrate in evaluating the optimal value for thickness of light water reflector using the neutron energy spectrum obtained at the core boundary. This is performed by simulating the neutron trajectory in the reflector in order to see its life end which is defined by one of the three following events:

- Neutron is backscattered from the reflector,

- Neutron is absorbed in the reflector,

- Neutron is leaked from the reflector.

Fig. 9 shows the obtained calculation results on neutron backscattering, absorption and leakage fractions as a function of the reflector thickness. In our consideration the optimal thickness of the reflector is selected from two evaluations: the first one is to determine the maximum value 


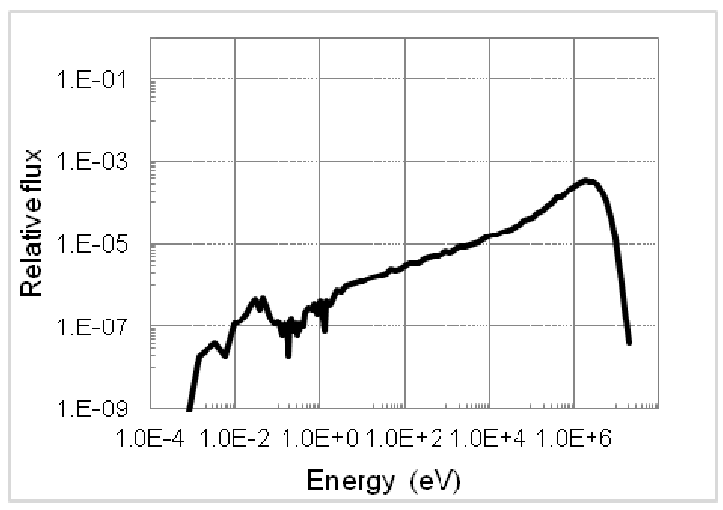

Fig. 8. Neutron energy spectrum at the fuel rod using the fission neutron source.

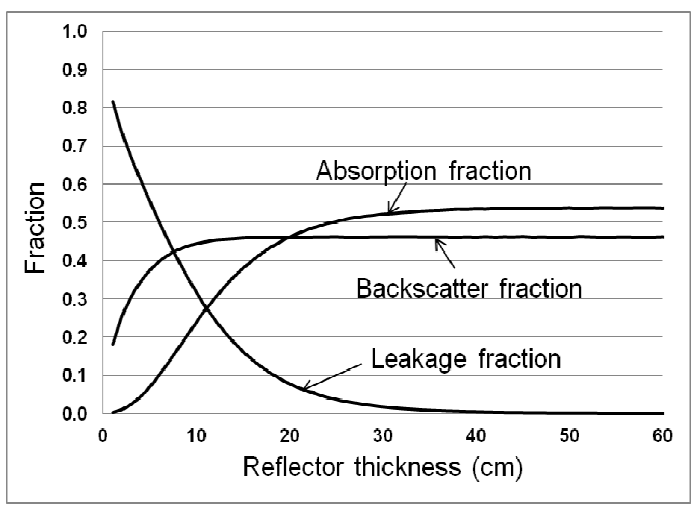

Fig. 9. Simulation results on neutron backscattering, absorption and leakage fractions as a function of the reflector thickness.

of the backscattering fraction, and the second one is based on the neutron shielding from leakage. Thus, from Fig. 9 we can see that for the first evaluation the reflector thickness satisfies at the value of more less $12 \mathrm{~cm}$, where the backscatter fraction becomes saturated. However, from the neutron shielding requirement based on evaluating the neutron leakage from the reflector to the containment the reflector thickness should be selected about $38-40 \mathrm{~cm}$, at which the neutron leakage is quite negligible. This evaluation result is well consistent with the design of AP-1000 reactor, a PWR type [7].

\section{CONCLUSION}

We have built a MC calculation program to consider the neutron transport in a typical configuration of the PWR fuel assembly, where the fission neutrons were assumed to generate at the fuel rods.

The validity of the simulation algorithms is confirmed by comparing our calculations with literature and MPNP code for the kinematic characteristics of the neutron transport.

The energy spectra of the neutrons hitting the fuel rod and the core boundary were obtained. The latter result was used to determine the optimum thickness of the light water reflector based on the concept of the backscattering fraction obtained by simulating the effects on neutron backscattering, absorption and leakage. The conclusion on the optimum thickness of about $38-40 \mathrm{~cm}$ is well consistent with the design of AP-1000 reactor.

\section{REFERENCES}

[1] John R. Lamarsh and Anthony J. Baratta, Introduction to Nuclear Engineering, Third Edition, Prince Hall 2001.

[2] HUST-Misubishi Collaboration program, Nuclear Power Plant Engineering -Text Book-Mitsubishi heavy industries, Ltd, 2011.

[3] Paul Reuss, Neutron Physics, EDP SCIENCES 2008.

[4] National Nuclear Data Center (NNDC), Brookhaven National Laboratory, http://www.nndc.bnl.gov.

[5] K. Bekurs and K. Virts, Neutron Physics, ATOMIZDAT 1968.

[6] T. Ueki, Lecture on Reactor Physics (JAEA-2013), Private publication.

[7] AP1000 European Design Control Document, EPS-GW-GL-700, Revision 1, Westinghouse. 